\title{
Research Paper: The Effect of a Nine-Weeks Training Program on The Center of Pressure Indicators With Open and Closed Eyes Condition in the Elderly Male
}

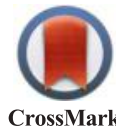

\author{
Ahmad Gol Rafati ${ }^{1},{ }^{*}$ Mansour Eslami ${ }^{1} \odot$, Shadmehr Mirdar ${ }^{1}$
}

1. Department of Physiology and Sport Biomechanics, Faculty of Physical Education and Sport Sciences, University of Mazandaran, Mazandaran, Iran.

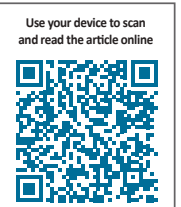

Crtat On: Rafati AG, Eslami M, Mirdar Sh. [The Effect of a Nine-Weeks Training Program on The Center of Pressure Indicators With Open and Closed Eyes Condition in the Elderly Male (Persian)]. Archives of Rehabilitation. 2018; 19(1):44-53. https:// doi.org/10.21859/JREHAB.19.1.44

https://doi.org/10.21859/JREHAB.19.1.44

Received: 10 Jul. 2017 Accepted: 13 Oct. 2017

Keywords:

Resistance training Center of pressure, Elderly, Static balance, Lower limbs

\section{ABSTRACT}

Objective Ageing is associated with some physiologic and functional declines that can increase disability, frailty, and falls in the elderly, so balance is used as a factor in determining the level of independence of the elderly. On the other hand, the mean velocity is considered as the most reliable indicator of the center of pressure to assess the balance and reduce the risk of falling. Because of significant effects of balance and muscle strength in the health of the more elderly population, the purpose this study was to investigate the effects of a nine- weeks training program on the center of pressure indicators with open and closed eyes condition in the elderly male.

Materials \& Methods In this study, 27 elderly subjects with general health were participated voluntarily in this study. These were randomly divided into two training groups $(n=15$, mean age $=61.5 \pm 08.59$ years, weight $=77.10 \pm 07.23 \mathrm{~kg}$, height $=167.6 \pm 6.4 \mathrm{~cm}$ ) and control $(\mathrm{n}=12$, mean age $=62.6 \pm 08.44$ years, weight $=72.07 \pm 08.06 \mathrm{~kg}$, height $=167.9 \pm 01.08 \mathrm{~cm}$ ). To assess the balance subjects were placed in a steady state on the foot scan at the beginning of the work, and the mean velocity of the center of pressure was first recorded in a static state with open eyes and then closed eyes. The kinetic data of the foot scan (mean velocity of the center of pressure) was calculated by RS-scan software, and the mean of the data was extracted from Excel software and evaluated. The training group then performed a nine-week program of lower limb muscle resistance training (on seven lower limb muscle groups including adductor and abductor of femurs, flexors, and extensor of femurs, flexors and extensor of knee, and plantar flexor of ankle) for 1.5 hour (15 minutes warm up, 1 hour of the main exercise, 15 minutes of cool down) and three sessions per week. During this period, the control group was requested to perform its daily activities. After completing the resistance training program, post-test was performed. Data were analyzed using independent and dependent T-analysis at a significant level $(\mathrm{P} \leq 0.05)$

Results Lower limb muscle resistance training for nine weeks yielded a significant improvement in mean velocity of $\operatorname{COP}(P=0.005, t=3.07)$ and static balance with open eyes $(P=0.004, t=3.46)$. In the training group, it has been pre-tested; however, no statistically significant effect was observed in static balance with closed eyes $(P=0.15, t=1.49)$. Resistance training showed a significant difference between the control and the training group in the mean overall velocity of the center of pressure $(P=0.04, t=2.43)$ and static balance with open eyes $(P=0.01, t=2.39)$ as determined by independent $t$-test. This difference was not significant between two groups in static balance with closed eyes ( $P=0.22, \mathrm{t}=1.25)$.

Conclusion The lower extremity muscle resistance training improves the center of pressure, static balance and reduces the fall risk. It is suggested that planning sufficient physical activities and training among all age groups is necessary. Appropriate resistance training program can prevent balance disorders and falling among the elderly population.

\section{* Corresponding Author:}

Mansour Eslami, PhD

Address: Department of Physiology and Sport Biomechanics, Faculty of Physical Education and Sport Sciences, Mazandaran University, Mazandaran, Iran.

Tel: +98 (915) 2145860

E-Mail: mseslami@gmail.com 


\title{
تأثير يك دوره برنامه تمرينهاى نههفتهاى بر شاخصهاى مركز فشار در حالت ايستاى ساكن با جشمان باز و بسته در مردان سالمند
}

\author{
احمدكل رفعتى'، "منصور اسلامى'ه، شادمهر ميردار' \\ ا - كروه فيزيولوريى و بيومكانيك ورزشى، دانشكده تربيتبدنى و علوم ورزشى، دانشكاه مازئندان، مازئدران، ايران.
}

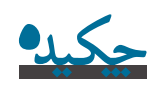

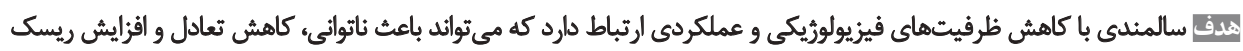

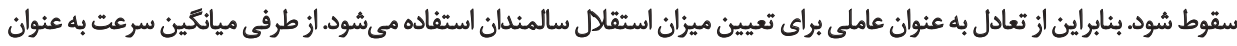

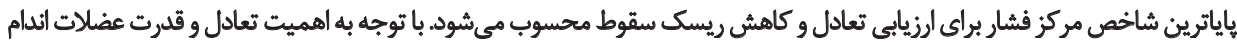

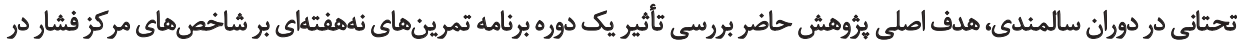

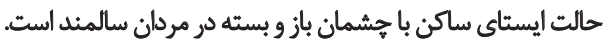

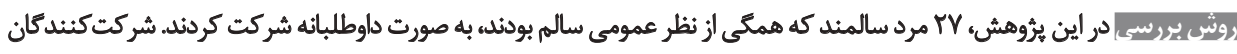

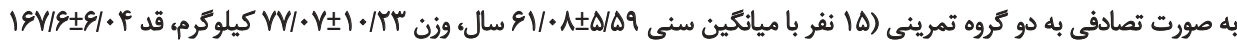

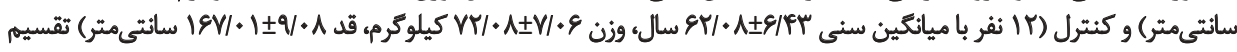

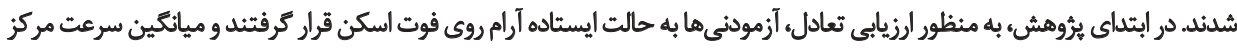

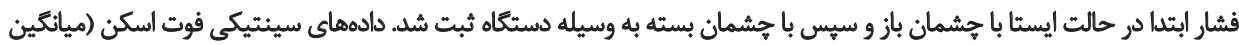

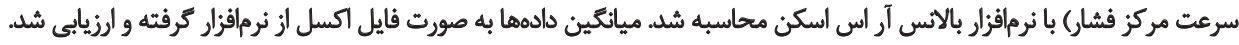

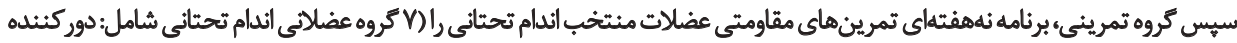

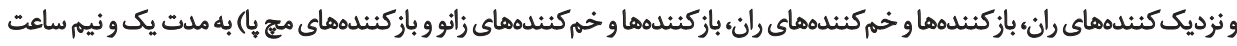

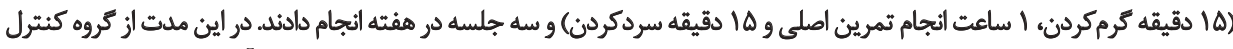

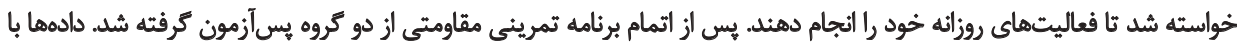

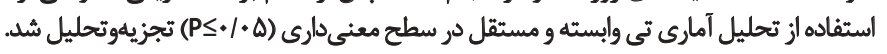

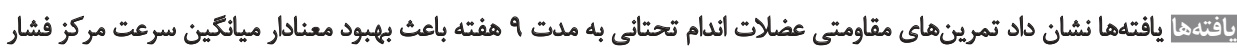

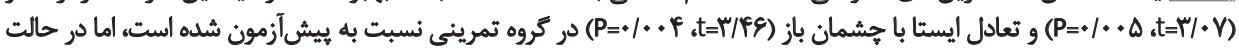

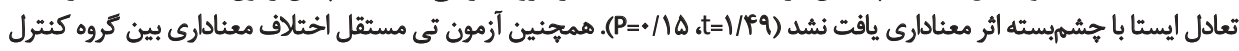

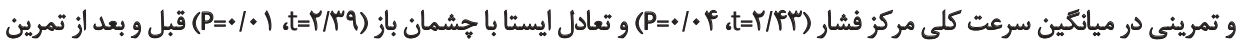

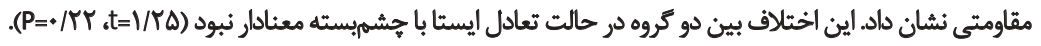

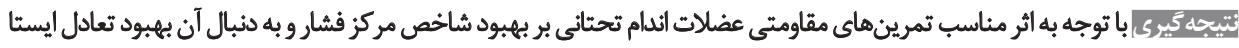

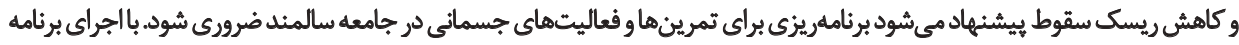

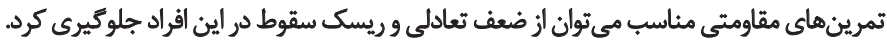

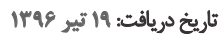

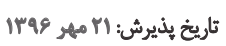

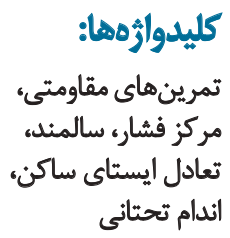

درصد از دو برابر فراتر خواهد رفت [r]. ازاينرو ييشعيرى از بروز

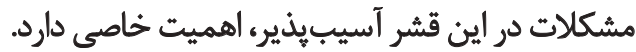

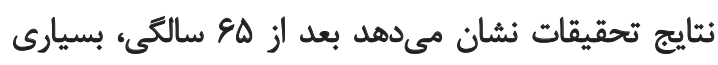

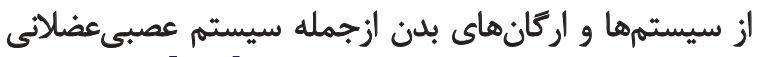

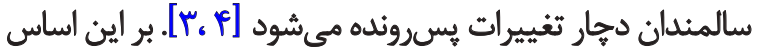

مقدمه

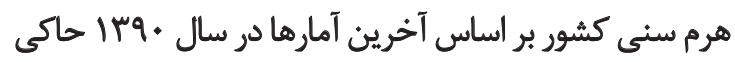

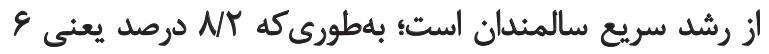

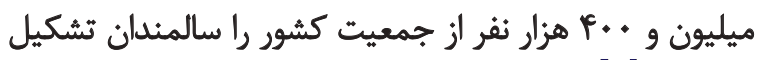

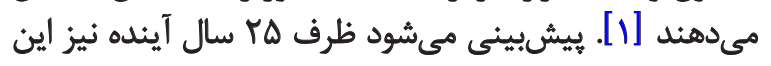

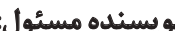
دكتر مينصور اسيلامي

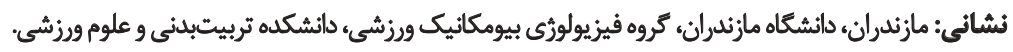
Pلفن: Pاني: رايانامه: mseslami@gmail.com 


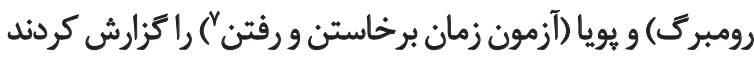

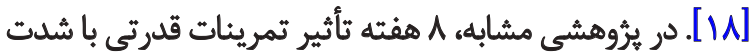

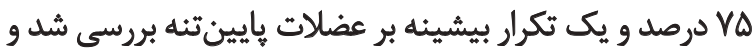

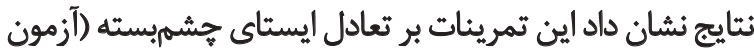

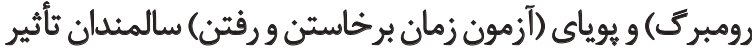

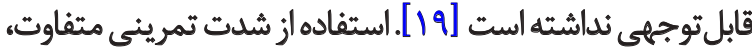

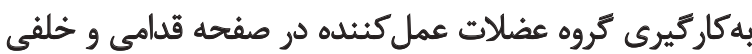

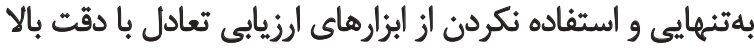

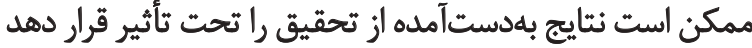
و سبب تناقض يافتهاي برُوهش شود است

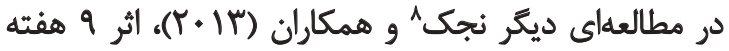

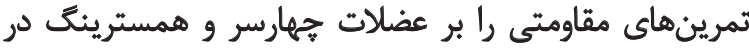

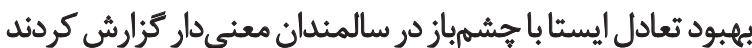

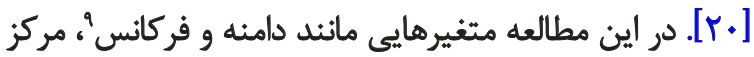

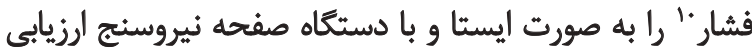

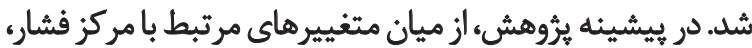

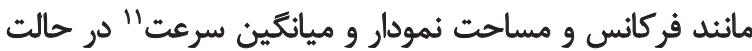

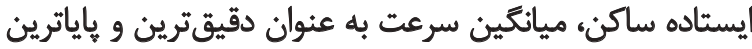

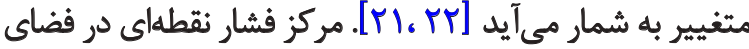

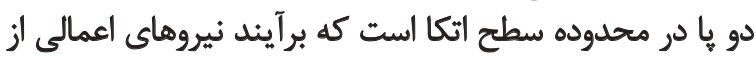

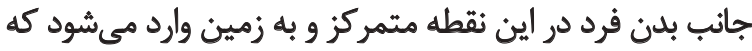

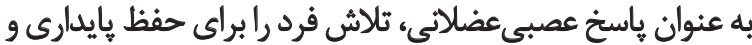

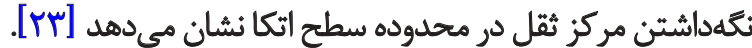
در يُروهشى اثر \& هفته تمرينهاى مقاومتى همسترينى،

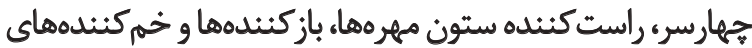

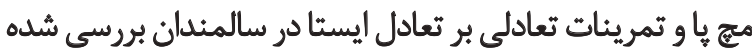

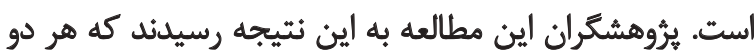

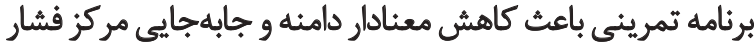

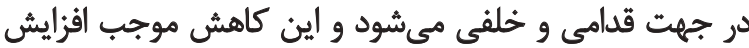
تعادل ميشود [Yrf]

در برنامه تمرينى مطالعات يادشده، به عضلات مهرم مانئد

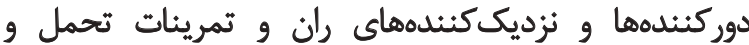

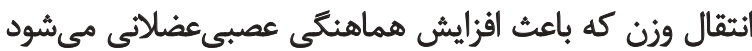

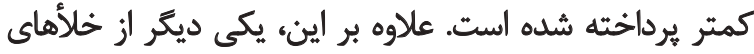

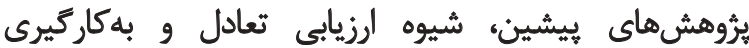

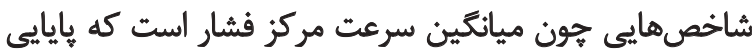

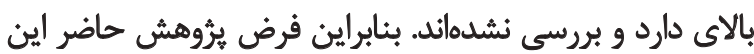

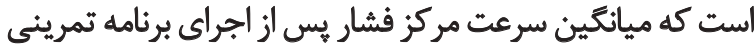

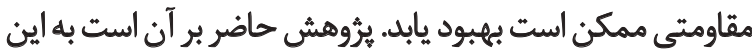

7. Time to go

8. Nejc

9. Amplitude \& frequency

10. Center of Pressure (COP)

11. Mean velocity
ريسك سقوط' يكى از مشكلات عمده سالمندى است [هاء؛

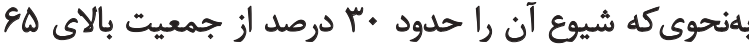

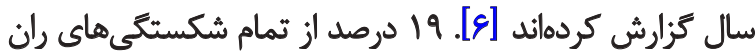

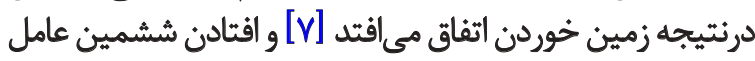
مركىمير در ميان سالمندان به شمار مىرود [A]

محققان در مطالعات متعدد، علل زمين خوردن سالمندان

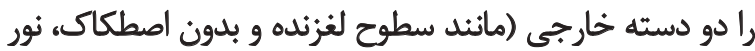

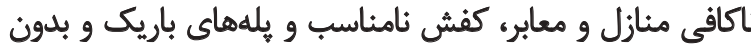

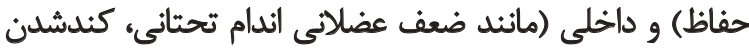

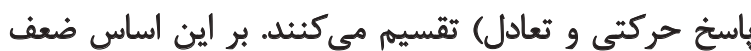

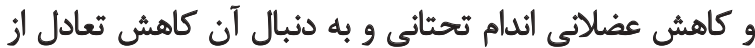

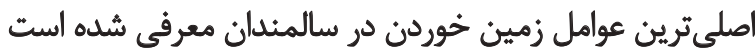

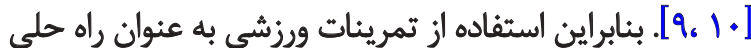

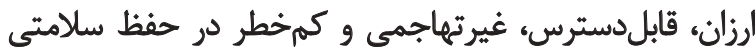

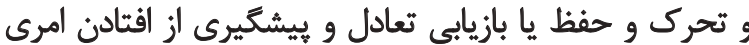

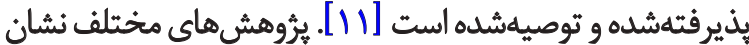

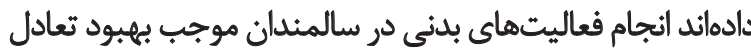

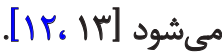

با در نظر گرفتن اين مداخلات، تمرين طراحى شده بايد شامل

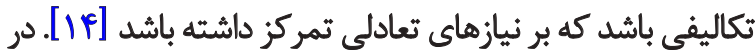

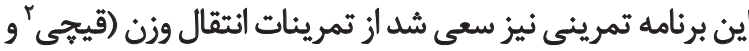

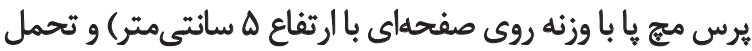

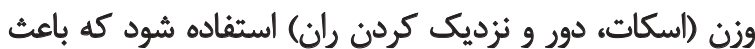

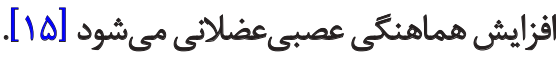

در مطالعات مشابه كه اثر برنامههاي قدرتي عضلات اندام

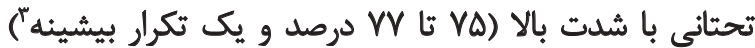

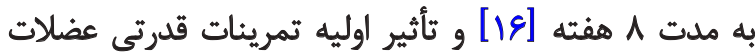

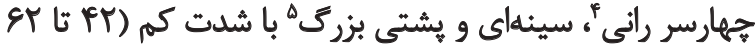

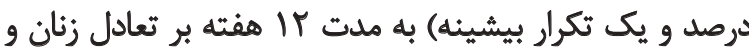

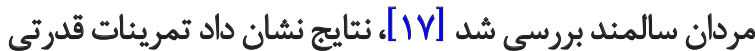

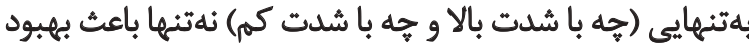

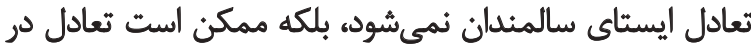
مردان سالمند رانيز كاهش دهد.

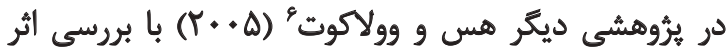

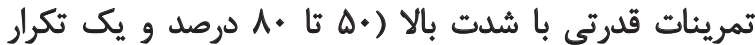

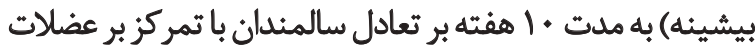
همسترينك و جهارسر، بهبود تعادل در برادل حالت ايستا (آزمون
1. Fall risk
2. Lunge
3. One Repetition Maximum (1-RM)
4. Quadriceps femoris
5. Pectoralis major and Latissimus dorsi
6. Hess \& Woollcott 
فوت اسكن، ميانكين سرعت مركز فشار (سه كوشش) ابتدا در

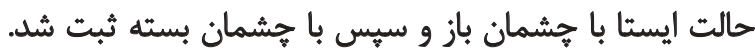

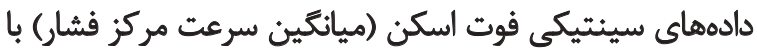

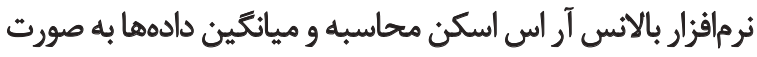

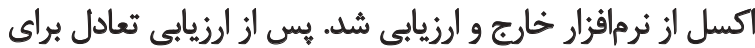

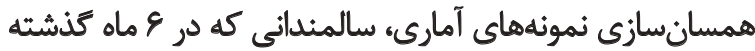

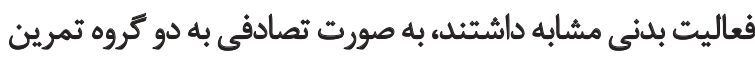

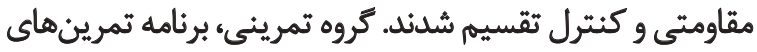

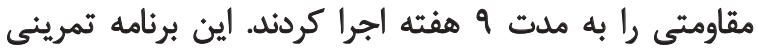

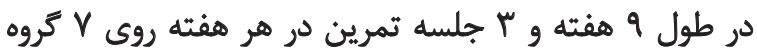

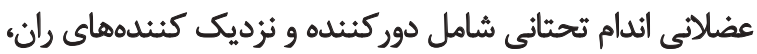

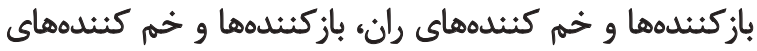

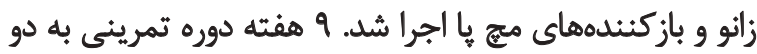

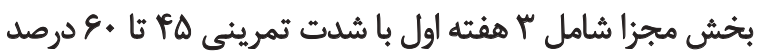

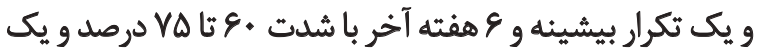

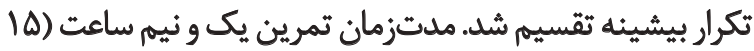

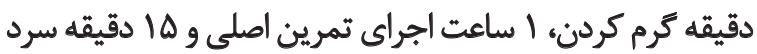

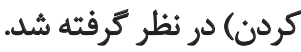

شدت تمرين از Fo درصد و يك تكرار بيشينه در هفتههاى

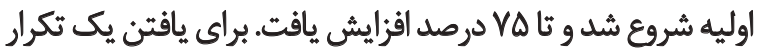

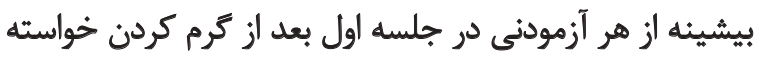

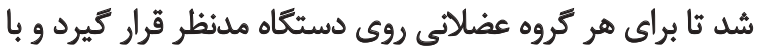

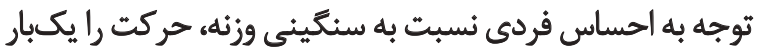

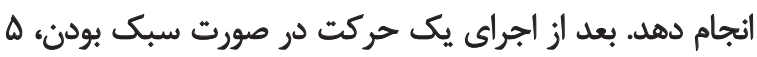

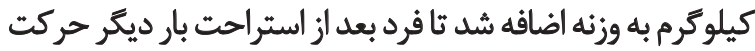

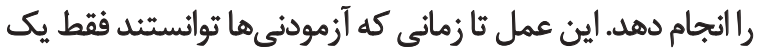

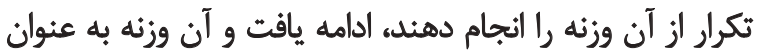

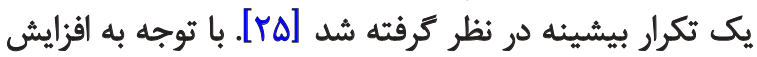

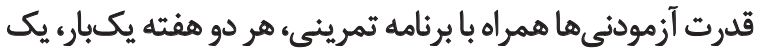

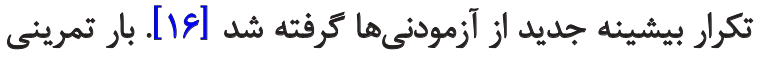

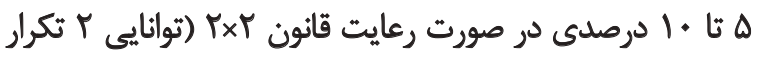

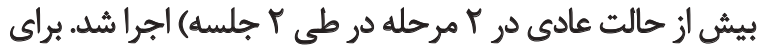

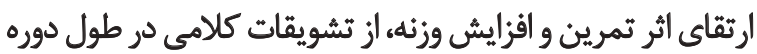

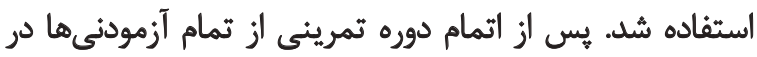

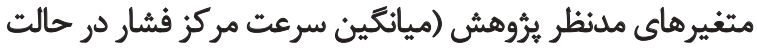
ايستا با جشمان باز و بسته و ميانكين كل سرعت مرعت مركز فشار)، در فر
هرسش ياسخ دهد كه برنامه تمرينى مقاومتى در حالت تحمل

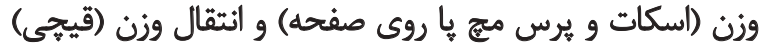

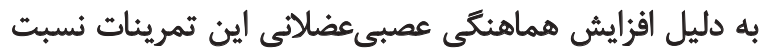

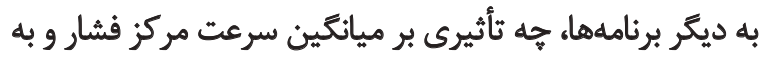
دنبال آن تعادل ايستاى سالمندان دارد.

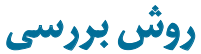

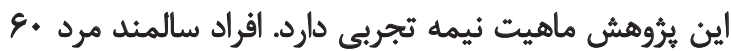

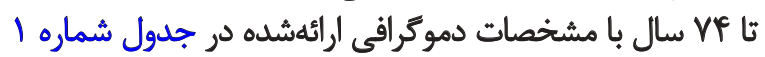

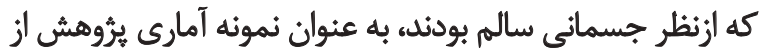

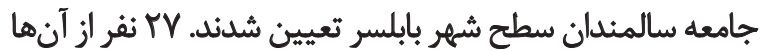

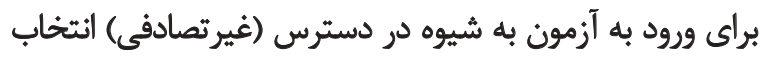

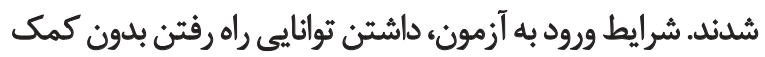

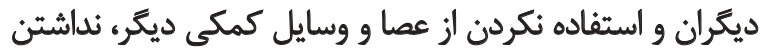

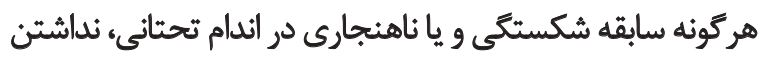

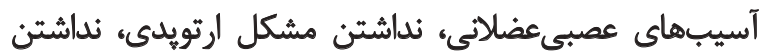

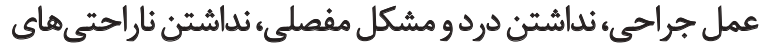

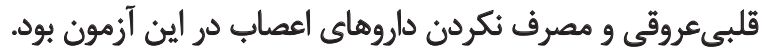

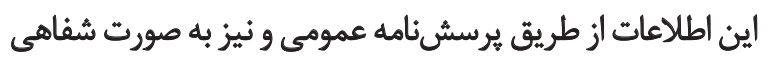

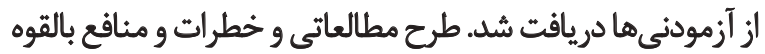

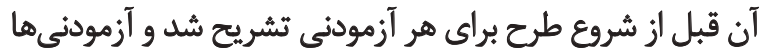
فرم رضايتنامه آكاهانه را تكميل و امضاك آردردئد. آبمنظور جمعآورى و ثبت اطلاعات ميانتين سرعت مركز فشار،

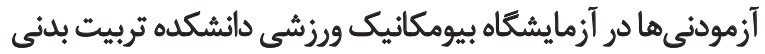

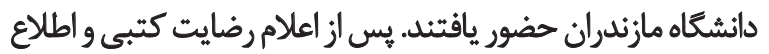

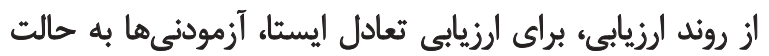

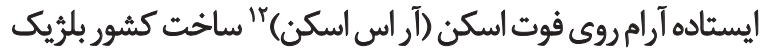

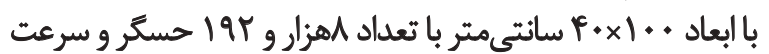

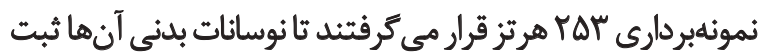

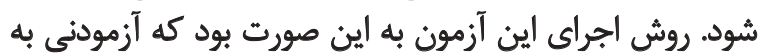

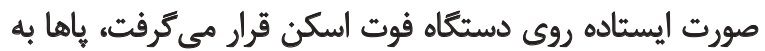

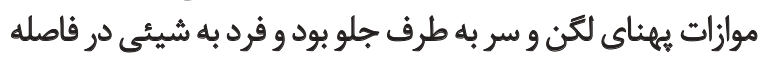

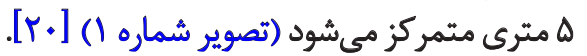
يّ از قراركرفتن آزمودنىها به حالت ايستاده آرام روى

12. RS-scan

جدولا. اطلاعات دموكرافى آزمودنىها (سن، وزن و قد)

\begin{tabular}{|c|c|c|c|c|}
\hline قد (ساتثىمتر) & وزن (كيلوكرم) & سن (سال) & تعداد & كروه \\
\hline $\mid Q V / F \pm \% / . F$ & $W / \cdot V \pm 1 \cdot / \pi$ & $8 / /+A \pm \Delta / \Delta 9$ & 10 & تمرينى \\
\hline $\mid 8 V /+1 \pm V / \cdot \lambda$ & $V /+\Lambda \pm V /+q$ & $R T /+A \pm g / R H$ & ir & كتترل \\
\hline
\end{tabular}

توانبخننى 


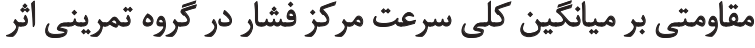

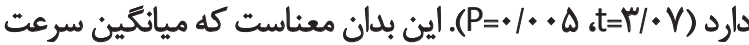

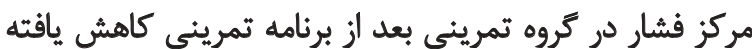

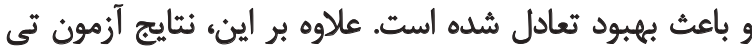

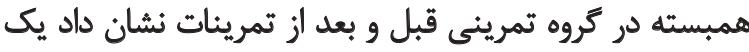

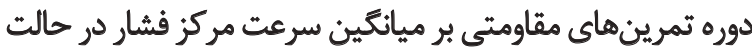

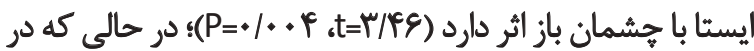

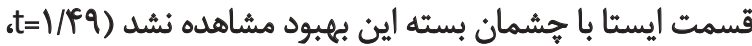

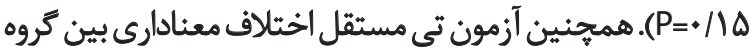

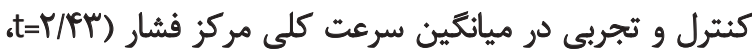

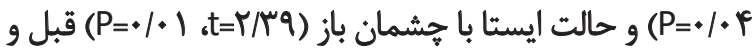

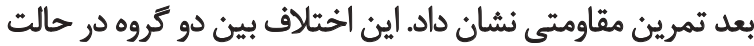

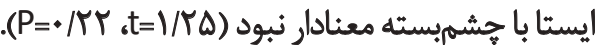

\&

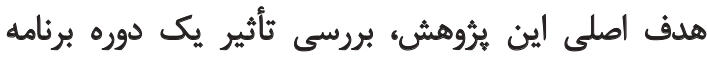

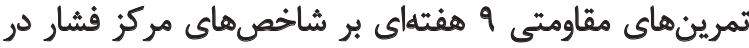

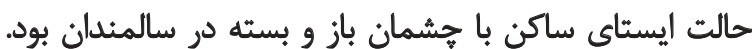

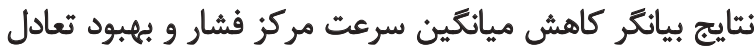

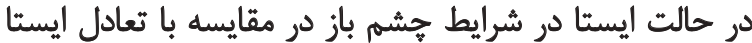

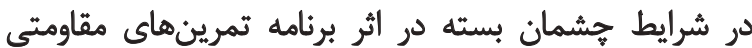

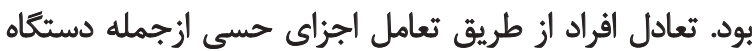

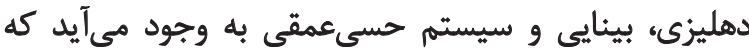

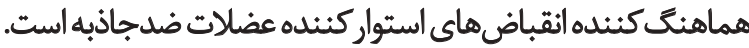

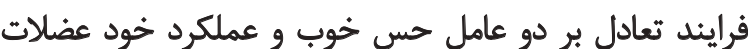

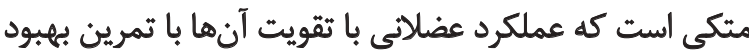

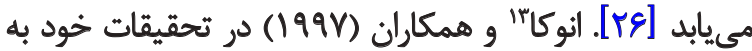
اين نتيجه رسيدند كه افزايش قدرت عضلانى دران اثر الثران تمرينات

13. Enoka

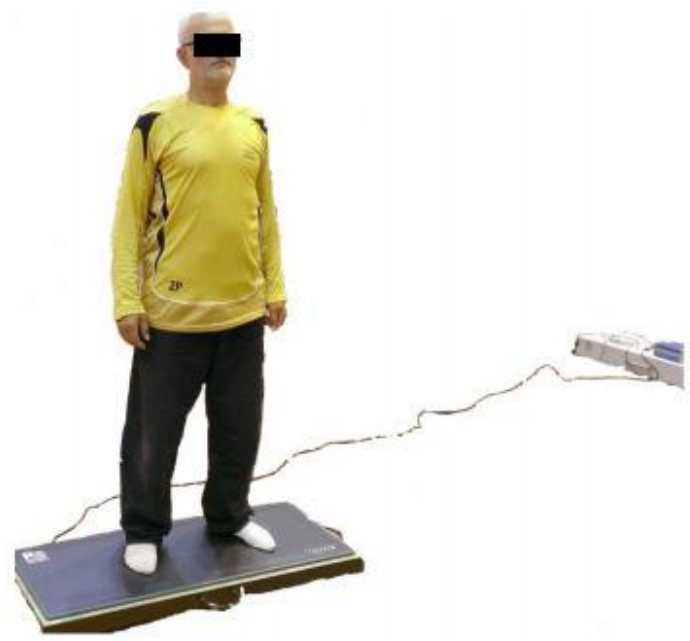

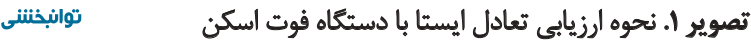
همان محيط و به همان روش بيشآزمونء بِ آزمون كرفته شد. بهمنظور استفاده از آزمون آمارى مناسب با توجه به حجم نمونيه

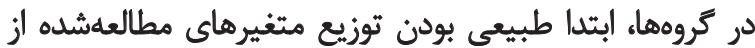

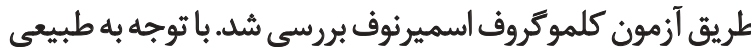

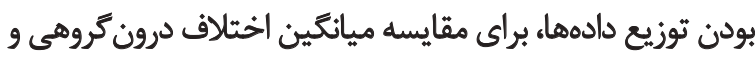

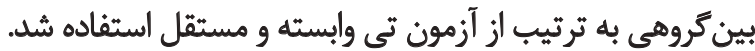

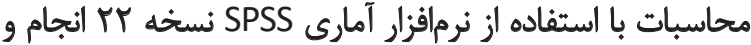

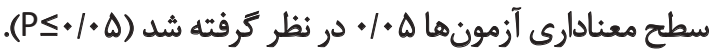

يافتهها

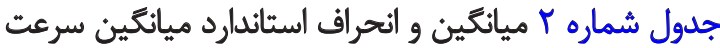

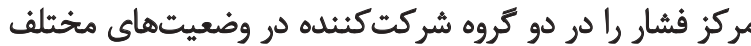

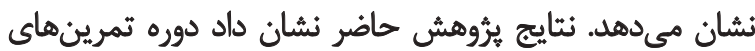

جدول Y. مثايسه ميانتيّين و انحراف استاندارد درون كروهى تمرينى و كنترل در زمان قبل و بعد از دوره تمرينى

\begin{tabular}{|c|c|c|c|c|c|}
\hline \multirow[b]{2}{*}{ 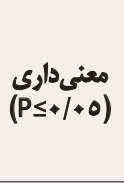 } & \multirow[b]{2}{*}{ 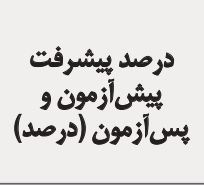 } & بعد & قبل & \multirow[b]{2}{*}{ شاخهص } & \multirow[b]{2}{*}{ كروهاها } \\
\hline & & (ميلمثراف ميانكين وائدارد & 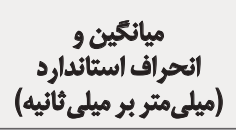 & & \\
\hline$\%$ & 19 & $g / N \pm$ W/YN & $V / \pm E Y / M \Delta$ & هيانكين سرعت جابهجايع مركز فشار & \\
\hline$\%$ & if & $\Delta / / \pm \varepsilon q / 1 r$ & $V / M \pm 1 / / r$ & تعادل در حالت ايستا با جششم باز & 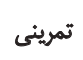 \\
\hline .110 & ir & $V / \Lambda \pm 1+1 \cdot r$ & $N T \pm 1 Q / 19$ & ثعادل در حالت ايستا با جشمه بسته & \\
\hline.$/ 9$. & $-* / * A$ & $V / \backslash \pm \mathscr{9} / 1 \Delta$ & $V / \backslash \pm \Delta q / / V$ & هيانكين سرعت جابهجايع هركز فشار & \\
\hline.$/ 94$ & -+198 & $V / / \pm 19 / M$ & $V / \cdot \pm 10 / 99$ & تعادل در حالت ايستا با جشه باز & 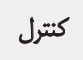 \\
\hline.$/ a r$ &.$- / \Delta T$ & $N \cdot \pm \mid r / q 4$ & $N \mid \pm * N Y$. & تعادل در حالت ايستا با يشهم بسته & \\
\hline
\end{tabular}




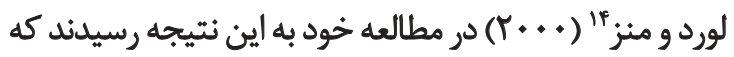

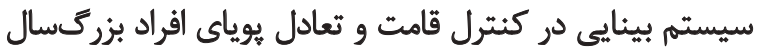

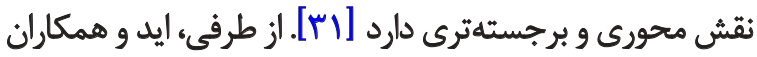

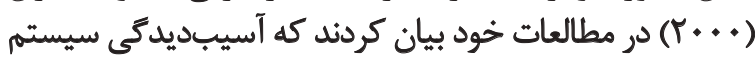

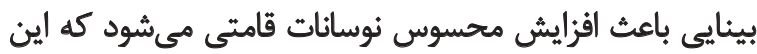

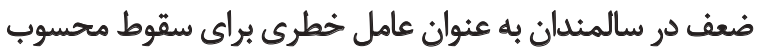

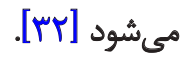

محققان در مطالعات خود به اين نتيجه رسيدند كه با بإنائ

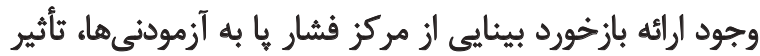

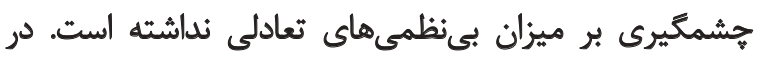

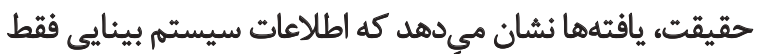

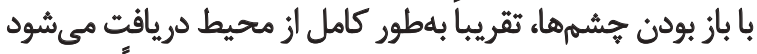

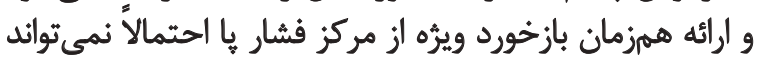

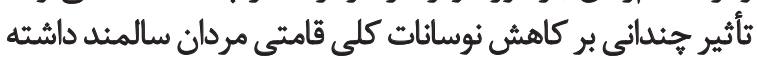

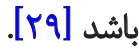

به نظر مىرسد سيستم بينايى در سازوكار تعادلى بلدن و

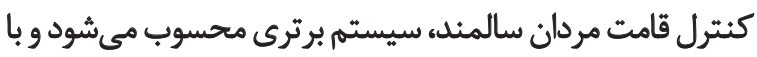

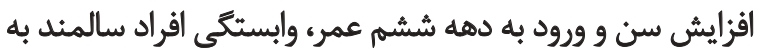

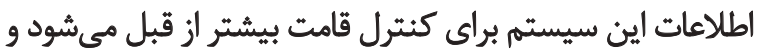

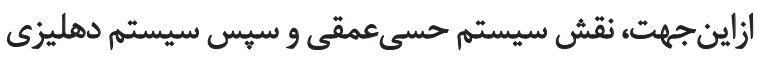

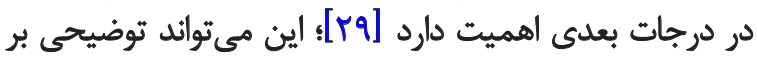

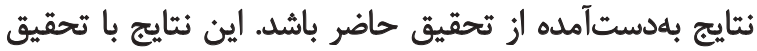

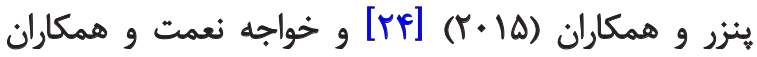

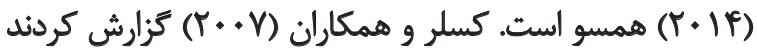

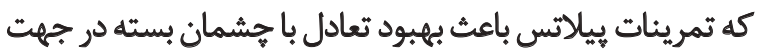

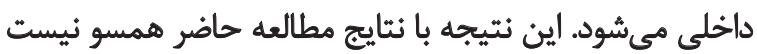

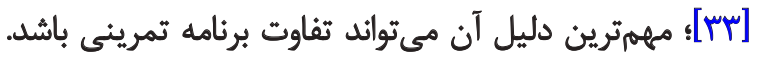

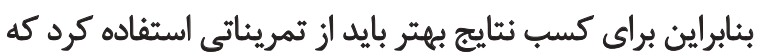
سيستمهاى تعادل رابيشتر دركير كند.

نتيجهيرى

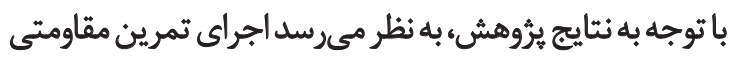

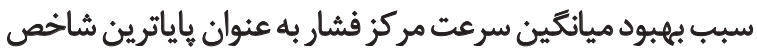

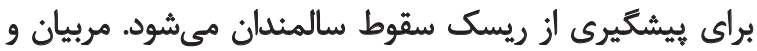

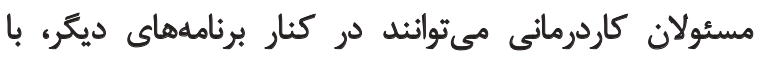

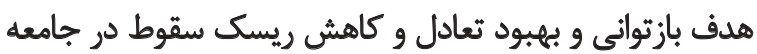

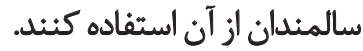

محدوديتهاى تحقيق حاضر شامل كنترل نشدن شرايط

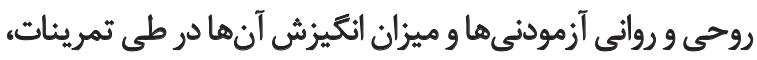

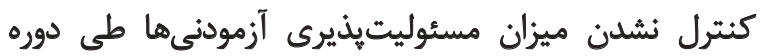
تمرينى، كنترل نشدن فعاليثهاى شبانهروزى و ميزان خواب و وني

14. Lord and Menz
مرتبط، ممكن است درنتيجه سازوكارهاى عصبى ازجمله افزايش

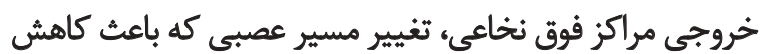

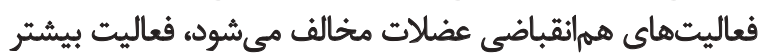

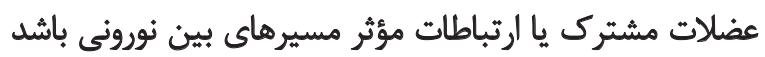

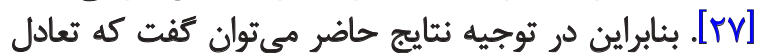

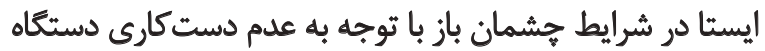

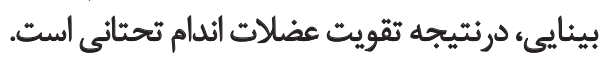

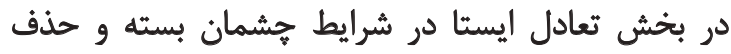

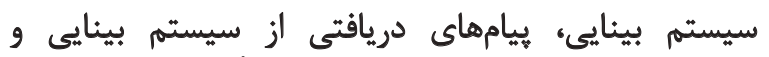

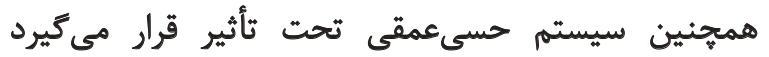

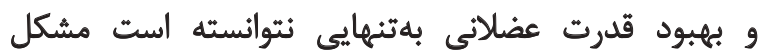

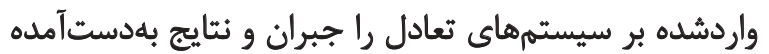

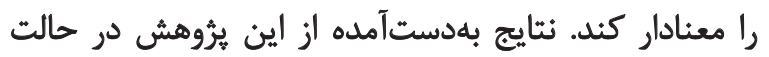

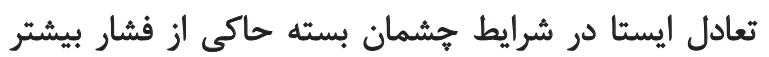

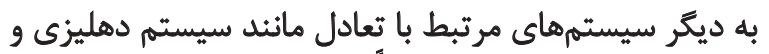

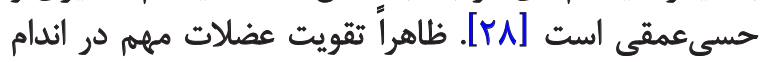

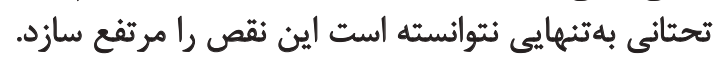

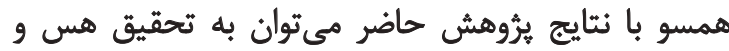

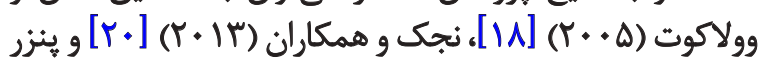

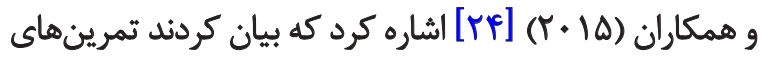

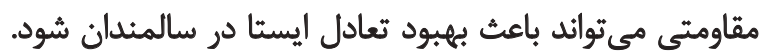

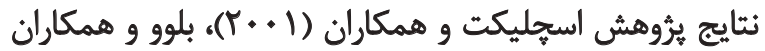

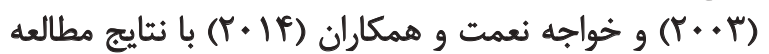

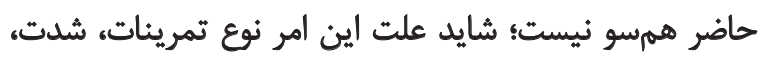
مدت دوره تمرينى، ابزارها و وسايل ارزيابى باشد.

نتايج ثرؤوهش نشان ميدهد تمرينهاى مقاومتى عضلات

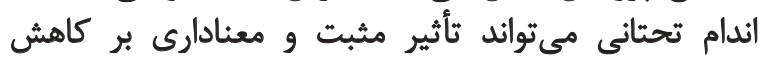

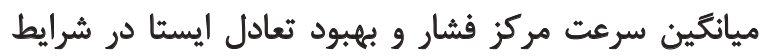

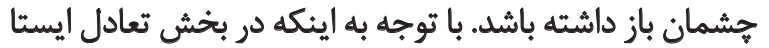

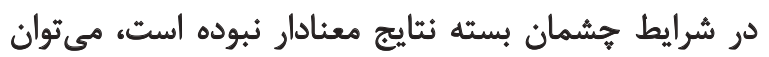

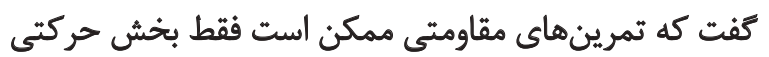

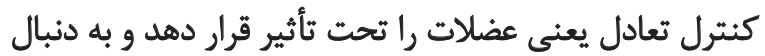

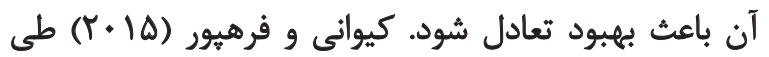

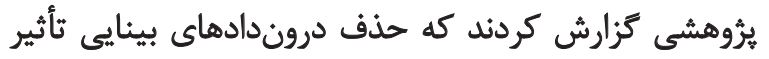

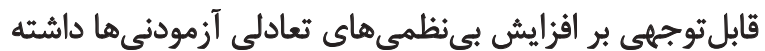

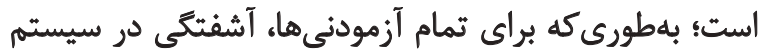

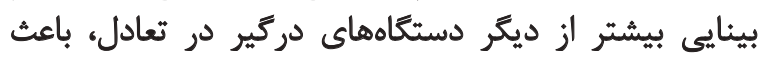

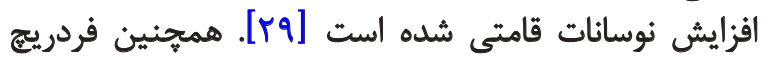

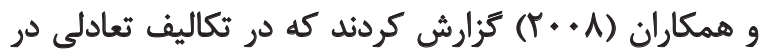

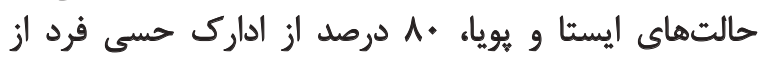

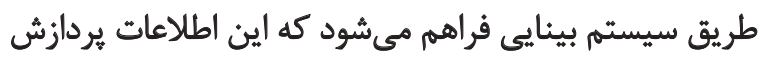

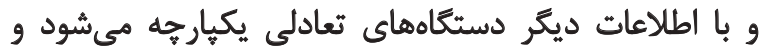

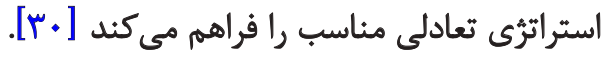


همجنين ميزان اثر تفاوتهاي فردى و ورائتى بر نتايج آزمون بود.

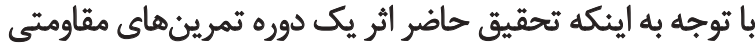

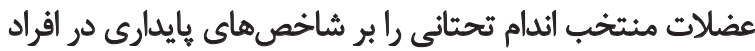

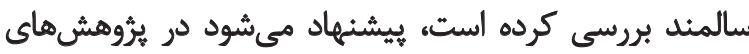

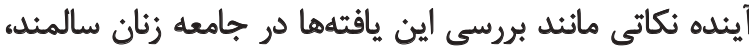

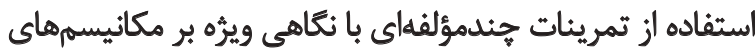

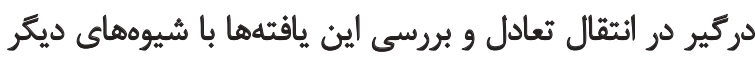

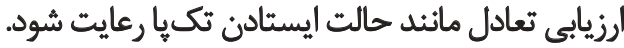

$$
\text { تشكر وقدردانى }
$$

اين مقاله از باياننامه مقطع كارشناسى ارشد آقاى احمدكل

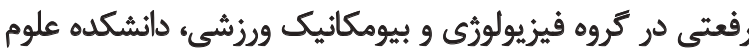

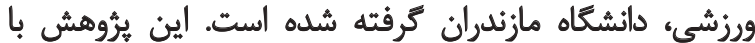

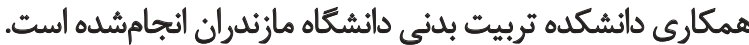

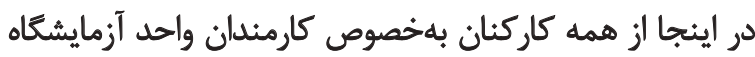

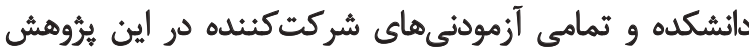
سياسكزارى مي كنيه. اين مقاله حامى مالي ندائي نداشته است. 


\section{References}

[1] Statistical Center of Iran. [Sellect result of the general gensus of population and housing (Persian)]. Tehran: Statistical Center of Iran; 2011.

[2] Evans D, Hodgkinson B, Lambert L, Wood J. Falls risk factors in the hospital setting: A systematic review. International Journal of Nursing Practice. 2001; 7(1):38-45. doi: 10.1046/j.1440172x.2001.00269.x

[3] Harada N, Chiu V, Damron Rodriguez J, Fowler E, Siu A, Reuben DB. Screening for balance and mobility impairment in elderly individuals living in residential care facilities. Physical Therapy. 1995; 75(6):462-9. doi: 10.1093/ptj/75.6.462

[4] Shumway Cook A, Baldwin M, Polissar NL, Gruber W. Predicting the probability for falls in community-dwelling older adults. Physical Therapy. 1997; 77(8):812-9. doi: 10.1093/ptj/77.8.812

[5] Woollacott M, Shumway Cook A. Attention and the control of posture and gait: A review of an emerging area of research. Gait \& Posture. 2002; 16(1):1-14. doi: 10.1016/s0966-6362(01)001564

[6] Fallah Pour M, Joghataei MT, A'shayeri H, Salavati M, Hosseini S A. [Effects of mental practice on balance in the elderly (Persian)]. Archives of Rehabilitation. 2003; 4(3-4):34-9.

[7] Park H, Kim KJ, Komatsu T, Park SK, Mutoh Y. Effect of combined exercise training on bone, body balance, and gait ability: a randomized controlled study in community-dwelling elderly women. Journal of Bone and Mineral Metabolism. 2008; 26(3):254-9. doi: 10.1007/s00774-007-0819-z

[8] Sattin R. Falls among older persons: A public health perspective. Annual Review of Public Health. 1992; 13(1):489-508. doi: 10.1146/annurev.publhealth.13.1.489

[9] Berg KO, Wood Dauphinee SL, Williams JI, Maki B. Measuring balance in the elderly: Validation of an instrument. Canadian Journal of Public Health= Revue Canadienne De Sante Publique. 1992; 83:S7-11. PMID: 1468055

[10] Shumway Cook A, Patla AE, Stewart A, Ferrucci L, Ciol MA, Guralnik JM. Environmental demands associated with community mobility in older adults with and without mobility disabilities. Physical Therapy. 2002; 82(7):670-81. PMID: 12088464

[11] Sadeghi H, Alirezaei F. [The effect of water exercise program on static and dynamic balance in elderly women (Persian)]. Salmand. 2008; 2(4):402-9.

[12] Justine M, Hamid TA, Mohan V, Jagannathan M. Effects of multicomponent exercise training on physical functioning among institutionalized elderly. ISRN Rehabilitation. 2012; 2012:1-7. doi: $10.5402 / 2012 / 124916$

[13] Nagai K, Yamada M, Tanaka B, Uemura K, Mori S, Aoyama T, et al. Effects of balance training on muscle coactivation during postural control in older adults: A randomized controlled Trial, The Journals of Gerontology Series A: Biological Sciences and Medical Sciences. 2012; 67(8):882-9. doi: 10.1093/gerona/glr252

[14] Cromwell RL, Meyers PM, Meyers PE, Newton RA. Tae kwon do: An effective exercise for improving balance and walking ability in older adults. The Journals of Gerontology Series A:
Biological Sciences and Medical Sciences. 2007; 62(6):641-6. doi: 10.1093/gerona/62.6.641

[15] Aagaard P, Suetta C, Caserotti P, Magnusson SP, Kjaer M. Role of the nervous system in sarcopenia and muscle atrophy with aging: strength training as a countermeasure. Scandinavian Journal of Medicine \& Science in Sports. 2010; 20(1):49-64. doi: 10.1111/j.1600-0838.2009.01084.x

[16] Schlicht J, Camaione DN, Owen SV. Effect of intense strength training on standing balance, walking speed, and sit-to-stand performance in older adults. The Journals of Gerontology Series A: Biological Sciences and Medical Sciences. 2001; 56(5):M281M286. doi: 10.1093/gerona/56.5.m281

[17] Bellew JW, Yates JW, Gater DR. The initial effects of lowvolume strength training on balance in untrained older men and women. Journal of Strength and Conditioning Research. 2003; 17(1):121-8. doi: 10.1519/00124278-200302000-00020

[18] Hess JA, Woollacott M. Effect of high-intensity strength-training on functional measures of balance ability in balance-impaired older adults. Journal of Manipulative and Physiological Therapeutics. 2005; 28(8):582-90. doi: 10.1016/j.jmpt.2005.08.013

[19] Khaje NK, Sadeghi H, Sahebozamani M. [The effect of 8 weeks of strength training on static and dynamic balance in older men (Persian)]. Sport Medicine (Harakat). 2014; 6(1):45-55.

[20] Nejc S, Loefler S, Cvecka J, Sedliak M, Kern H. Strength training in elderly people improves static balance: A randomized controlled trial. European Journal of Translational Myology. 2013; 23(3):85. doi: 10.4081/bam.2013.3.85

[21] Moghadam M, Ashayeri H, Salavati M, Sarafzadeh J, Taghipoor $\mathrm{KD}$, Saeedi A, et al. Reliability of center of pressure measures of postural stability in healthy older adults: Effects of postural task difficulty and cognitive load. Gait \& Posture. 2011; 33(4):651-5. doi: 10.1016/j.gaitpost.2011.02.016

[22] Qiu H, Xiong S. Center-of-pressure based postural sway measures: Reliability and ability to distinguish between age, fear of falling and fall history. International Journal of Industrial Ergonomics. 2015; 47:37-44. doi: 10.1016/j.ergon.2015.02.004

[23] Winter DA. Biomechanics and motor control of human movement. New Jersey: John Wiley \& Sons; 2009 Oct 12.

[24] Penzer F, Duchateau J, Baudry S. Effects of short-term training combining strength and balance exercises on maximal strength and upright standing steadiness in elderly adults. Experimental Gerontology. 2015; 61:38-46. doi: 10.1016/j.exger.2014.11.013

[25] Henwood TR, Riek S, Taaffe DR. Strength versus muscle power-specific resistance training in community-dwelling older adults. The Journals of Gerontology: Series A. 2008; 63(1):83-91. doi: $10.1093 /$ gerona $/ 63.1 .83$

[26] Butler AA, Lord SR, Rogers MW, Fitzpatrick RC. Muscle weakness impairs the proprioceptive control of human standing. Brain Research. 2008; 1242:244-51. doi: 10.1016/j.brainres.2008.03.094

[27] Enoka RM. Neural strategies in the control of muscle force. Muscle \& Nerve. 1997; 20(S5):66-9. Available from: https://doi. org/10.1002/(sici)1097-4598(1997)5+<66::aid-mus16>3.3.co; 2-d

[28] Bellew JW, Fenter PC, Chelette B, Moore R, Loreno D. Effects of a short-term dynamic balance training program in healthy old- 
er women. Journal of Geriatric Physical Therapy. 2005; 28(1):4-8. doi: 10.1519/00139143-200504000-00001

[29] Kiyani P, Farahpoor N. [Evaluation of performance of the vestibular proprioception and vision systems on postural control of old men (Persian)]. Iranian Journal of Ageing. 2015; 10(3):44-53.

[30] Friedrich M, Grein H-J, Wicher C, Schuetze J, Mueller A, Lauenroth A, et al. Influence of pathologic and simulated visual dysfunctions on the postural system. Experimental Brain Research. 2007; 186(2):305-14. doi: 10.1007/s00221-007-1233-4

[31] Lord SR, Menz HB. Visual contributions to postural stability in older adults. Gerontology. 2000; 46(6):306-10. doi: $10.1159 / 000022182$

[32] Eid L, Ferrario V, Sforza C. Sensorial afferents and center of foot pressure in blind and sighted adults. Journal of Visual Impairment \& Blindness (JVIB). 2000; 94(02).

[33] Kaesler DS, Mellifont RB, Kelly PS, Taaffe DR. A novel balance exercise program for postural stability in older adults: A pilot study. Journal of Bodywork and Movement Therapies. 2007; 11(1):37-43. doi: 10.1016/j.jbmt.2006.05.003 
